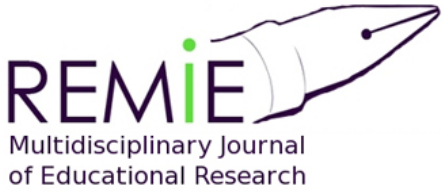

Multidisciplinary Journa of Educational Research

\section{Hipatia Press}

www.hipatiapress.com

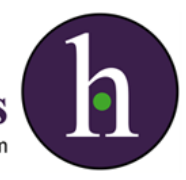

Instructions for authors, subscriptions and further details:

http://remie.hipatiapress.com

\title{
Efecto de la Escolarización en la Producción de Relaciones Causales y Contracausales: Evidencia Psicolingüística para el Ámbito Educativo
}

Gabriela Mariel Zunino ${ }^{1,2}$

1) Consejo Nacional de Investigaciones Científicas y Técnicas (CONICET). Argentina

2) Facultad de Filosofía y Letras, Universidad de Buenos Aires (UBA). Argentina.

Date of publication: October $15^{\text {th }}, 2016$

Edition period: October 2016 - February 2017

To cite this article: Zunino, G. M. (2016). Efecto de la Escolarización en la Producción de Relaciones Causales y Contracausales: Evidencia Psicolingüística para el Ámbito Educativo. Multidisciplinary Journal of Educational Research, 6(3), 258-291. doi: 10.17583/remie.2016.2127

To link this article: $h t t p: / / d x . d o i . o r g / 10.17583 /$ remie.2016.2127

\section{PLEASE SCROLL DOWN FOR ARTICLE}

The terms and conditions of use are related to the Open Journal System and to Creative Commons Attribution License (CCAL). 


\section{Formal Education Effect on the Production of Causal and Counter-causal Relations: Psycholinguistic Evidence for the Educational Field}

Gabriela Mariel Zunino

CONICET/UBA-Argentina

\section{Abstract}

In order to promote the practical application of psycholinguistic data in educational fields and expecting that this transfer would enhance the development of both the pedagogical field and the investigation in experimental psycholinguistics, we present two experiments to analyse the production of semantic relations in discourse, especially the causality/countercausality dimension. We found that the pattern of causal advantage is cross-wise and consistent in subjects with different levels of formal education, so it could be a suitable scaffold to develop other aspects of discourse comprehension and production. We compare our results with previous findings about discourse comprehension and interpret the data in the framework of educational processes. To use of empirical evidence about language processing on educational fields allows not only to review specific issues such as the characteristics of teaching materials, but also to improve educational process in a comprehensive way, making possible to adapt different approaches to populations with different characteristics.

Keywords: psycholinguistics, semantic relations, causality, education 


\section{Efecto de la Escolarización en la Producción de Relaciones Causales y Contracausales: Evidencia Psicolinguiística para el Ámbito Educativo}

Gabriela Mariel Zunino

CONICET/UBA-Argentina

\section{Resumen}

Con el objetivo de propiciar la aplicación concreta de datos psicolingüísticos en ámbitos educativos y de que esta transferencia funcione para potenciar el desarrollo tanto de los espacios pedagógicos como de investigación en psicolingüística experimental, presentamos dos experimentos que estudian la producción de relaciones semánticas en los discursos, especialmente, la dimensión de causalidad/contracausalidad. Hallamos que el patrón de ventaja causal es transversal y consistente en sujetos con distinto nivel de escolarización formal, por lo que podría resultar un adecuado andamiaje para desarrollar otros aspectos de la comprensión y producción de discursos. Comparamos nuestros resultados con hallazgos anteriores en procesos de comprensión de discursos e interpretamos los datos en el marco de los procesos educativos. La utilización de la evidencia empírica sobre procesamiento de lenguaje permite no solo revisar cuestiones específicas como las características de los materiales didácticos, sino mejorar el proceso pedagógico de modo global, en función de adecuar los modos de abordaje a poblaciones con características disímiles.

Palabras clave: psicolingüística, relaciones semánticas, causalidad, educación 
1 problema de la causalidad ha sido abordado por múltiples disciplinas, desde la filosofía y la psicología hasta la lingüística en sus variados enfoques. Sin embargo, no muchos de los estudios han intentado articular abordajes de índole general con su aplicación concreta y específica en diversos ámbitos cotidianos como la educación (Cheng, 1997; Couper-Kuhlen \& Kortman, 2000; Davidson, 1985; Goldman, Graesser \& van den Broek, 1999; Goldvarg \& Johnson-Laird, 2001; Hume, 1740; Kant, 1787/2008; Kim, 2007; Leslie, 1994; McNamara, Kintsch, Songer \& Kintsch, 1996; Michotte, 1963; Pérez, 1999; Schlottmann, 2001; Sloman, 2005; Sperber, Premack \& Premack, 1995; Strawson, 1985; Viale, 1999; Waldmann, 2001; entre otros). Asimismo, el estudio psicolingüístico sobre el procesamiento de relaciones semánticas en los discursos -tanto orales como escritos- ha sido abordado por distintas investigaciones, pero pocas han reflexionado sobre cuáles son las posibilidades de transferencia de la evidencia empírica obtenida, en función de aplicar los hallazgos, por ejemplo, en ámbitos educativos con el objetivo de mejorar los procesos de alfabetización en distintos niveles y espacios de escolarización.

Una de las líneas de investigación que ha abordado este problema, a veces tangencialmente, es la que se ha encargado de estudiar cómo impacta la experticia $-y$, por ende, la posibilidad de hacer intervenir conocimientos previos sobre una determinada temática- durante la comprensión de textos (McNamara et al. 1996; Ozuru, Dempsey \& McNamara, 2009; van Dijk y Kintsch, 1983; Zunino, 2014, 2016; Zwaan, Magliano \& Graesser, 1995; entre otros). Se han analizado factores propios de los materiales lingüísticos como el tipo de información y el tipo textual, además de variables relativas a los sujetos como el nivel de escolarización o el ámbito de conocimiento especializado de los mismos. Si bien la mayoría de los estudios sobre comprensión de textos $\mathrm{y}$, especialmente, sobre relaciones causales se realizaron con textos narrativos, existen algunos que desplazan la mirada a textos expositivos y fue en ese movimiento que se vio con mayor claridad la necesidad de involucrar otra variable fundamental en el proceso: el conocimiento de mundo del lector ${ }^{1}$ (Zunino, 2014, 2016; Dery \& Koenig, 2015; Graesser, McNamara \& Louwerse 2003; León \& Peñalba, 2002; Martins, Kigiel \& Jhean-Larose, 2006; McNamara et al. 1996; Noordman, 
Vonk \& Kempff, 1992; Noordman \& Vonk, 1998; Noordman, Vonk, Cozijn \& Frank, 2015; Ozuru et al., 2009; Raiter, 2000; Xiang \& Kuperberg, 2015; entre otros). Si bien no existe evidencia concluyente sobre cuál es el efecto de los conectores y de la marcación explícita de las relaciones semánticas/conceptuales en los textos expositivos -esto es, si el impacto es similar o muestra el mismo patrón que en textos narrativos-, estos estudios han demostrado con mayor claridad que: a) el efecto del conocimiento de mundo es decisivo -sobre todo en el nivel de representación más abstracto: el modelo de situación ${ }^{2}-$ b) existe una articulación de modo permanente e inevitable con las marcas lingüísticas presentadas por el texto; c) las estrategias de procesamiento puestas en juego por lectores expertos y no expertos son significativamente distintas. El tipo específico de interacción que presenta la información lingüística explícita y el conocimiento previo sobre el mundo, no obstante, sigue en discusión (Degand \& Sanders, 2002; Kendeou \& van den Broek, 2007; McNamara et al., 1996; Noordman \& Vonk, 1998).

Otra cuestión central en este panorama ha sido la escasez de trabajos sobre producción de discurso y el análisis de los potenciales puntos en común o de contraste respecto de procesos de comprensión (Mar, 2004). En trabajos anteriores (Zunino, 2014, 2016; Zunino \& Raiter, 2012), hemos analizado procesos de comprensión: planteamos y respaldamos lo que denominamos "Hipótesis de causalidad por defecto" -también defendida por Sanders (2005) con algunas diferencias específicas-. De modo general, esta hipótesis plantea que las relaciones causales son esperadas y establecidas como estrategia por defecto cuando un sujeto procesa discurso y que, por ende, su procesamiento (psico)lingüístico será más sencillo -más preciso y más rápido- que el de otras relaciones semánticas. Asimismo, las relaciones causales presentarán menos requerimientos de marca semántica explícita -como las partículas conectivas- para ser adecuadamente comprendidas. Por otro lado, hemos analizado de modo preliminar parte de los datos sobre producción presentados en este artículo en otro trabajo reciente (Zunino, 2016) como primer acercamiento al proceso de producción de discurso y, específicamente, al papel de la dimensión causal/contracausal en él. Aquí intentaremos articular estos datos previos 


\section{Zunino - Causalidad y Contracausalidad}

con nuevos resultados para analizar esa interacción en función de utilizar esta evidencia más específicamente en el espacio educativo.

En términos de cómo estos datos pueden aportar estrategias relevantes en el ámbito de la educación, es especialmente importante notar que: a) una ventaja causal transversal a los niveles de escolarización -esto es, la posibilidad de construir causalidad sin especial entrenamiento- puede resultar un andamiaje ideal para desarrollar la producción y comprensión de discursos/textos y proyectar esta facilidad a otras relaciones semánticas que sí requieran entrenamiento especial; b) el bajo rendimiento durante la comprensión de textos con una ostensible ausencia de marcas semánticas explícitas, especialmente en temáticas no familiares o desconocidas, no indica necesariamente dificultades del sujeto -en este caso, del estudiante-, sino del material linguístico que se le ofrece -por ejemplo, los textos del material didáctico utilizado-, por lo que un especial cuidado sobre este aspecto en la selección y diseño de materiales didácticos puede repercutir en una mejora significativa de su comprensión.

En este trabajo pretendemos aportar evidencia convergente para procesos de construcción y producción de relaciones causales y contracausales. En este sentido, nuestro objetivo es sumar datos sobre tareas de producción en un contexto de escasa evidencia empírica ${ }^{3}$, y analizar de qué modo estos resultados pueden ser utilizados, en conjunto con hallazgos anteriores, para pensar estrategias que mejoren la comprensión y producción de discursos en el ámbito educativo. Presentamos dos experimentos que implican tareas de producción y se complementan entre sí: uno de ellos plantea un completamiento guiado por opciones múltiples y el otro intenta analizar el proceso de completamiento espontáneo sin más guía o restricción semántica que la partícula conectiva. Por otra parte, el primero presenta sólo una estructura linguística posible para cada tipo de relación: consecutivas con "entonces" para las relaciones causales y adversativas con "pero" para las relaciones contracausales. El segundo experimento, en cambio, permite ver qué sucede con otro tipo de estructuras lingüísticas que también verbalizan relaciones causales y contracausales: causales invertidas con "porque" y concesivas con "aunque". Relacionar los resultados en ambos casos nos dará una idea más cabal del proceso analizado. 


\section{Experimento 1}

Este experimento tiene como objetivo verificar si la "Hipótesis de causalidad por defecto" se evidencia y de qué modo lo hace cuando la tarea propuesta implica comprender un fragmento textual inconcluso -presentado en dos condiciones: sin y con partícula conectiva- y seleccionar, de entre cuatro opciones, un final adecuado que complete el texto de modo coherente. Nuestra predicción es que, si esta hipótesis se cumple efectivamente, los lectores seleccionarán con mayor frecuencia y en menor tiempo las opciones de completamiento causal, incluso sin que haya marcas explícitas como partículas conectivas causales y sin distinciones según el nivel de escolarización formal del sujeto.

\section{Método}

\section{Participantes}

A partir de un muestreo aleatorio estratificado por nivel de escolarización formal, se conformaron dos grupos: alta escolaridad y baja escolaridad. En el grupo de alta escolaridad (AE), participaron 50 sujetos, con un promedio de 40,2 años de edad, D.E.13,79, hablantes nativos de español rioplatense, con una escolarización formal de entre 12 y 18 años -promedio $=17,32$; D.E. $=1,53-$. En el grupo de baja escolaridad (BE), participaron 34 sujetos de ambos sexos, con un promedio de edad de 28,82 años, D.E. 12,67, hablantes nativos de español rioplatense, con una escolarización formal de entre 7 y 12 años -promedio 10,54 años; D.E. $=0,9-{ }^{4}$.

Los datos se trataron como medidas repetidas -diseño de emparejamiento de sujetos o matched subject (Gravetter \& Wallnau, 2009). Organizados en parejas, uno de los integrantes de la pareja realizó la tarea en la condición sin partícula conectiva y el otro realizó la tarea con partícula conectiva presente. Se obtuvieron datos de 25 participantes de alta escolaridad y 17 de baja escolaridad por condición evaluada. 


\section{Materiales}

Fueron diseñados 18 textos breves $^{5}$ que se encontraban inconclusos, seguidos por 4 opciones de completamiento: opción causal (C), opción contracausal (CC), opción coherente sin relación causal (sin RC), opción incongruente/inconsistente (INC). Todos los textos fueron controlados en su extensión por cantidad de palabras: entre 30 y 42 palabras por texto, con un promedio de 34,6 .

Las extensiones de las opciones de respuesta fueron controladas por cantidad de palabras del siguiente modo: la suma de las palabras contenidas por las cuatro frases en cada ítem siempre fue de entre 15 y 25 palabras y su promedio de 20,9 palabras por ítem. Si se consideran los dos tipos de estímulos generados por la inclusión de la partícula conectiva, surge lo siguiente: los estímulos causales presentan un promedio de 22 palabras por grupo de opciones; los estímulos contracausales presentan un promedio de 20 palabras por grupo de opciones.

Se controló el orden de presentación de las opciones: la opción causal aparecía 4 veces en primer, tercer y cuarto lugar y 5 veces en segundo lugar; el resto de las opciones variaban entre 4 y 6 veces en cada posición, en función de la ubicación de la opción causal en cada caso.

En la primera condición evaluada, se presentaron los 18 textos sin partícula conectiva: en este caso, la elección no estaba guiada explícitamente. La segunda condición evaluada presentó los mismos 18 textos, pero se incluyeron partículas conectivas que marcaban explícitamente la relación semántica esperada y guiaban el completamiento: la mitad de los estímulos se presentaron con partícula causal "entonces" y la otra mitad, con partícula contracausal "pero".

En relación con la estructura sintáctica de las oraciones utilizadas y otras restricciones gramaticales, debe aclarase que: 1) tenían una estructura básica $\mathrm{S}-\mathrm{V}-\mathrm{O}$; 2) se armaron oraciones breves, evitando oraciones compuestas; 3) los verbos siempre se presentaron en modo indicativo y variaron entre presente y pasado -se usan ambos tipos de pretéritos simples, según el estímulo-; 4) no se presentaron estructuras hendidas, proposiciones incluidas adjetivas -ni especificativas ni explicativas-, proposiciones incluidas adverbiales o proposiciones incluidas sustantivas -excepto en un unos pocos 
casos de discurso referido simple: "le dijo que" o "le pidió que" en función de que respetaran la variedad de español de los hablantes que iban a realizar las tareas-; 5) se evitaron todas las negaciones explícitas, tanto de los estímulos como de las preguntas: se utilizaron sólo negaciones léxicas cuando resultaba estrictamente necesario.

Ejemplo de estímulos utilizados:

(1) Javier hace atletismo desde chico. Siempre le gustó correr, disfruta mucho de hacer actividad física. Desde que empezó a competir internacionalmente, debe entrenar todos los días varias horas. (entonces/pero) Su rendimiento deportivo...

1.- es mucho mejor que hace unos años. (C)

2.- genera alegría en otros deportistas. ( $\sin \mathrm{RC}$ )

3.- profesor de matemática. (INC)

4.- sigue igual que hace unos años. (CC)

\section{Procedimiento}

Las pruebas fueron programadas en SuperLab $4.0^{6}$. Se evaluó tanto la adecuación o el tipo de respuesta como los tiempos de lectura del estímulo (TRL) y de respuesta o resolución de la tarea (TRR). La administración de la prueba fue individual con el evaluador presente.

En todos los casos, se presentó la consigna por escrito en la pantalla de la computadora y oralmente por parte del evaluador. Luego de cada consigna, los sujetos realizaban un ejemplo de práctica con el fin de verificar la adecuada comprensión del ejercicio.

El experimento constaba de una lista de 20 ítems -18 target y 2 fillers al inicio de cada bloque para propiciar la habituación a la tarea- en dos condiciones: sin partícula conectiva específica y con partícula conectiva causal o contracausal, lo que generaba 9 ítems para cada dimensión semántica. Cada participante estuvo frente a un bloque de 20 ítems, presentados al azar.

Para comenzar con el experimento, el participante debía presionar la barra espaciadora. Inmediatamente aparecía un texto escrito en letras negras sobre pantalla blanca; el sujeto debía leer con atención el fragmento y luego presionar nuevamente la barra espaciadora. En esa instancia, aún con el texto presente, aparecían las 4 opciones de completamiento numeradas del 1 al 4 y 
el participante debía elegir qué opción creía que completaba mejor el fragmento que había leído. Si creían que ninguna completaba adecuadamente el fragmento, podían presionar la barra espaciadora y seguir con el siguiente texto.

\section{Resultados}

En primer término, se llevó a cabo un análisis exploratorio que permitiera detectar los casos extremos de TR y depurar la base de datos ${ }^{7}$. Se optó por utilizar un método de detección dependiente del tamaño muestral (Cousineau \& Chartier, 2010; Thompson, 2006). Se calcularon las medias y los desvíos de cada sujeto en cada condición o para cada respuesta. Para la primera condición, se consideraron las medias de TRs para elecciones causales vs. no causales. Para la segunda condición se consideraron los TRs de ítems respondidos adecuadamente para cada modalidad -causal vs. contracausal-. Los datos eliminados a partir de seguir el método de Van Selst y Jolicoeur (1994) implicaron todos aquellos resultados que quedaran por fuera de 2 desvíos estándar (DE) de la media por sujeto por condición: a) primera condición, en el grupo AE: se eliminó el 5\% de los datos para los TRL y el $3 \%$ para los $\mathrm{TRR}^{8}$, b) primera condición grupo BE: se eliminó 4\% de los TRL y el 5\% de los TRR; c) segunda condición, grupo AE: se eliminó el 4\% de los TRL y el 4\% de los TRR; d) segunda condición, grupo BE: se eliminó el 2\% de los TRL y el 3\% de los TRR. En todas las condiciones, se decidió reemplazar los casos extremos por el valor de la media de cada sujeto en cada condición (Ratcliff, 1979, 1993).

Para el análisis del tipo de respuesta -niveles de respuestas correctas/adecuadas- con análisis de varianzas -con porcentajes o proporciones no es posible realizar este tipo de prueba (Woods, Fletcher \& Hughes, 1986)-, se realizó una transformación logística sobre la proporción de respuestas de cada tipo o adecuadas -según la condición- de cada sujeto.

\section{Condición sin partícula conectiva}

Para detectar efectos principales e interacciones entre las variables, realizamos un análisis factorial completo ${ }^{9}$ con un factor independiente o 
entre sujetos -ESCOLARIZACIÓN- y un factor intra-sujetos o medidas repetidas -TIPO DE COMPLETAMIENTO: causal vs, no causal-. Para el tipo de respuesta, la variable de TIPO DE COMPLETAMIENTO -causal vs. no causal- mostró un efecto principal significativo $(\mathrm{F}(1,35)=259,88 ; \mathrm{p}=, 000)$ y también fue significativa su interacción con el factor de ESCOLARIZACIÓN $(\mathrm{F}(1,35)=13,5 ; \mathrm{p}=, 001)$. Para los valores de TRL, sólo la interacción TIPO DE COMPLETAMIENTO*ESCOLARIZACIÓN $(\mathrm{F}(1,33)=4,94 ; \mathrm{p}=, 033)$ resultó significativa; mientras para los TRR sólo se obtuvo efecto principal de TIPO DE COMPLETAMIENTO $(\mathrm{F}(1,33)=13,14$; $\mathrm{p}=, 001)$.

Tabla 1

Experimento 1: tipos de completamiento, tiempos de lectura y respuesta (ms) y desvíos estándar en condición sin partícula.

\begin{tabular}{llllll}
\hline & & Rta\% & $\begin{array}{c}\text { Rta TLog } \\
(\mathrm{DE})\end{array}$ & TRL (DE) (ms) & \multicolumn{1}{c}{ TRR (DE) (ms) } \\
\hline \multirow{3}{*}{ AE } & Causal & 92,5 & $2,18(, 687)$ & $9714,02(2257,59)$ & $9233,11(3966,69)$ \\
& No causal & 7,3 & $-2,39(, 562)$ & $11382,52(2862,81)$ & $14867,62(10426,16)$ \\
& Causal & 77,7 & $1,40(, 836)$ & $13936,22(6042,87)$ & $11912,13(7247,12)$ \\
BE & No causal & 21,0 & $-1,47(, 806)$ & $13487,56(5880,58)$ & $13937,66(8719,52)$ \\
\hline
\end{tabular}

Elaboración propia. $\mathrm{AE}=$ Alta Escolaridad; $\mathrm{BE}=\mathrm{Baja}$ Escolaridad; ms=milisegundos; TLog=resultado de transformación logística de porcentajes; TRL=tiempo de lectura; TRR=tiempo de respuesta; DE=desvío estándar.

Para el nivel de respuesta, el contraste entre completamientos causales vs. no causales fue estadísticamente significativo en ambos grupos de participantes $(\mathrm{AE}: \mathrm{F}(1,19)=307,4 ; \mathrm{p}=, 000$ y $\mathrm{BE}: \mathrm{F}(1,16)=52,54 ; \mathrm{p}=, 000)$ a favor de los completamientos causales. Para TRL, en cambio, sólo existió un efecto estadísticamente significativo para el grupo $\mathrm{AE}(\mathrm{F} 1(1,18)=9,42$; $\mathrm{p}=, 007 ; \mathrm{F} 2(1,11)=15,49 ; \mathrm{p}=, 002)$. Para TRR, el grupo AE mostró un contraste significativo a favor del completamiento causal $(\mathrm{F} 1(1,18)=10,66$; $\mathrm{p}=, 004 ; \mathrm{F} 2(1,11)=7,93 ; \mathrm{p}=, 017)$, mientras que para el grupo BE la diferencia entre las medias sólo fue significativa para F2 $(\mathrm{F} 1(1,15)=3,75 ; \mathrm{p}=, 072$; $\mathrm{F} 2(1,16)=13,74 ; \mathrm{p}=, 002)$. 
Finalmente, se realizaron los contrastes entre grupos según nivel de escolarización, con ANOVA para muestras independientes. Sólo la variable tipo de completamiento -causal vs. no causal- mostró diferencias significativas entre los grupos, a favor de los sujetos de alta escolaridad. Para completamiento causal: $\mathrm{F}(1,40)=10,21 ; \mathrm{p}=, 003$. Para completamiento no causal: $\mathrm{F}(1,36)=16,47 ; \mathrm{p}=, 000$. Las medias de TRL y TRR no se distinguieron significativamente según el nivel de escolarización de los sujetos.

\section{Condición con partícula conectiva}

El análisis factorial completo, con un factor entre sujetos ESCOLARIZACIÓN- y un factor intra-sujetos -DIMENSIÓN SEMÁNTICA: causal vs. contracausal- mostró un efecto principal de DIMENSIÓN SEÁNTICA para la precisión de respuestas $(\mathrm{F}(1,40)=25,31$; $\mathrm{p}=, 000)$ y una interacción significativa entre DIMENSIÓN SEMÁNTICA*ESCOLARIZACIÓN $\quad(\mathrm{F}(1,40)=4,29 ; \mathrm{p}=, 045)$. Para la medida de TRL no se encontraron ni efectos principales ni interacciones significativas. Para los TRR, en cambio, se halló un efecto principal significativo del factor intra-grupo de DIMENSIÓN SEMÁNTICA $(\mathrm{F}(1,40)=55,63 ; \mathrm{p}=, 000)$.

\section{Tabla 2}

Experimento 1: respuestas adecuadas, tiempos de lectura y respuesta (ms) y desvios estándar con partícula conectiva.

\begin{tabular}{llcllll}
\hline & & $\begin{array}{l}\text { RtaAd } \\
\text { ec \% }\end{array}$ & $\begin{array}{l}\text { Rta } \\
\text { (DE) }\end{array}$ & TLog & TRL (DE) (ms) & TRR (DE) (ms) \\
\hline AE & Causal & 92,3 & $4,72(3,10)$ & $11052,72(2593,37)$ & $9355,65(3480,64)$ \\
& Contracausal & 81,1 & $3,13(3,23)$ & $11352,28(3260,24)$ & $15181,48(6158,41)$ \\
BE & Causal & 86,9 & $3,67(3,05)$ & $13958,06(3863,69)$ & $10525,16(4282,12)$ \\
& Contracausal & 47,1 &,$- 150(1,01)$ & $13686,62(4206,58)$ & $17850,61(9397,28)$ \\
\hline
\end{tabular}

Elaboración propia. $\mathrm{AE}=$ Alta Escolaridad; $\mathrm{BE}=\mathrm{Baja}$ Escolaridad; $\mathrm{ms}=$ =milisegundos; TLog=resultado de transformación logística de porcentajes; TRL=tiempo de lectura; TRR=tiempo de respuesta; $D E=$ desvío estándar. 


\section{REMIE - Multidisciplinary Journal of Educational Research 269}

Tabla 3

Experimento 1: completamientos inadecuados según tipo de estímulo: opciones elegidas.

\begin{tabular}{llccccc}
\hline & & $\mathrm{Rta} C(\%)$ & $\mathrm{RtaCC}(\%)$ & $\operatorname{SinRC}(\%)$ & $\mathrm{Inc}(\%)$ & $\mathrm{N} / \mathrm{R}(\%)$ \\
\hline \multirow{4}{*}{$\mathrm{AE}$} & $\mathrm{C}$ & ---- & 20 & 66,7 & 6,7 & 6,7 \\
& $\mathrm{CC}$ & 89,5 & ---- & 7,9 & 2,6 & ---- \\
& $\mathrm{C}$ & ---- & 27,8 & 61,1 & 11,1 & ---- \\
$\mathrm{BE}$ & $\mathrm{CC}$ & 87,3 & ---- & 1,4 & 7,0 & 4,2 \\
\hline
\end{tabular}

Elaboración propia. $\mathrm{AE}=\mathrm{Alta}$ escolaridad; $\mathrm{BE}=\mathrm{Baja}$ escolaridad; $\mathrm{C}=$ ítems causales; $\mathrm{CC}=$ ítems contracausales; Rta $\mathrm{C}=$ completamiento causal; Rta $\mathrm{CC}=$ completamiento contracausal; $\mathrm{Sin}$ $\mathrm{RC}=$ completamiento sin relación causal/contracausal; Inc=completamiento inconsistente; $\mathrm{N} / \mathrm{R}=$ no responde.

Para el contraste entre estímulos causales y contracausales dentro de cada grupo de sujetos, la diferencia en los niveles de precisión de respuestas para ambas dimensiones fue significativa en ambos grupos a favor de los ítems causales $(\mathrm{AE}: \mathrm{F}(1,24)=4,35 ; \mathrm{p}=, 048$ y $\mathrm{BE}: \mathrm{F}(1,16)=33,53 ; \mathrm{p}=, 000)$. Las medidas de TRL no mostraron diferencias significativas en ninguno de los dos grupos, pero el contraste de medias de TRR fue estadísticamente significativo en el grupo AE también con ventaja causal: $F 1(1,24)=36,4$; $\mathrm{p}=, 000 ; \mathrm{F} 2(1,17)=11,02 ; \mathrm{p}=, 004$. Para el grupo $\mathrm{BE}$, en cambio F1 resultó significativa, pero $F 2$ no lo fue: $F 1(1,16)=20,88 ; p=, 000 ; F 2(1,17)=3,55$; $\mathrm{p}=, 078)$.

Además, se hicieron los contrastes entre grupos a través de pruebas ANOVA para muestras independientes. Para la precisión de respuestas, la dimensión causal no mostró diferencias significativas entre los grupos, pero sí fue significativo el contraste para la dimensión contracausal $(F(1,40)=16,25 ; p=, 000)$. Inversamente, para TRL, sólo fueron significativas las diferencias en la dimensión causal $(\mathrm{F} 1(1,40)=8,54 ; \mathrm{p}=, 006$; F2 $(1,17)=14,65 ; \mathrm{p}=, 001)$. Para TRR, la causales mostraron diferencias significativas para $\mathrm{F} 1 \quad(\mathrm{~F} 1(1,40)=4,09 ; \mathrm{p}=, 050)$, pero no para $\mathrm{F} 2$ $(\mathrm{F} 2(1,17)=2,37 ; \mathrm{p}=, 143)$. 


\section{Discusión}

En principio, es necesario decir que la tarea propuesta implica, por supuesto, la comprensión del fragmento, pero, además, requiere construir una relación semántica en pos de generar un texto globalmente coherente. En este sentido, la prueba permite evaluar el proceso activo ${ }^{10}$ de construcción de relaciones de significado que los lectores llevan adelante durante la comprensión y resulta especialmente informativa para verificar si la causalidad efectivamente es una relación preferida durante ese proceso.

En ambos grupos de sujetos, existió una tendencia marcada por el completamiento causal y una diferencia estadísticamente significativa respecto de los completamientos no causales. Este primer dato respalda de modo claro nuestra hipótesis de causalidad por defecto (Zunino, 2014, 2016). Esto es: en caso de no haber ninguna marca explícita sobre la relación semántica a establecer, el lector se inclina por construir una relación causal. A partir de este mismo dato, un segundo punto importante es la consistencia en el patrón de respuestas entre ambos grupos de sujetos. Sin bien en el grupo de baja escolaridad el porcentaje de elección causal decreció levemente, el patrón de ventaja causal continúa siendo marcado. Si se mira en detalle, en el grupo de baja escolaridad, lo que se da es una mayor dispersión de las elecciones: hay más casos de "no responde" y una dispersión mayor en la elección de otras opciones, incluso la opción considerada de completamiento "inconsistente". Si bien este dato parece evidenciar que la comprensión general resulta más difícil para el grupo con menor escolarización formal -esto es, la pericia en la comprensión se encuentra condicionada por la escolarización-, esta variable no parece condicionar lo que podríamos denominar "patrón causal". Este dato es central para nuestros objetivos: si existe evidencia de que las relaciones causales son básicas y fundantes para la organización de nuestras representaciones mentales sobre el mundo y sobre los discursos -sin intervención de la escolarización-, este patrón podría usarse como estructura conceptual y discursiva de sostén para abordar pedagógicamente tanto la comprensión y producción de textos como habilidad general, como el manejo específico de otros aspectos lingüísticos y discursivos no susceptibles de procesamiento por defecto. 
En la condición con partícula conectiva presente es posible notar que el patrón de preferencia causal se mantiene para ambos grupos de sujetos. Los ítems causales tienen un nivel de completamientos adecuados significativamente mayor que los contracausales, en ambos grupos de sujetos. Más aún, en el grupo de baja escolaridad, el porcentaje de completamientos adecuados en ítems contracausales es menor al 50\%. Este dato es de por sí informativo, y se refuerza con el análisis acerca de qué tipo de elección hacen los sujetos en los casos en que no eligen la respuesta adecuada en relación con la restricción semántica impuesta por el conector. En ambos grupos existe un patrón similar. Para estímulos causales, las elecciones inadecuadas están sistemáticamente desplazadas hacia la opción semánticamente plausible fuera de la dimensión causal/contracausal. Esto parece implicar una suerte de debilitamiento de la relación semántica: serían casos en que la guía lingüística no parece ser lo suficientemente fuerte y sólo llega a propiciar un completamiento semántico consistente pero más débil o menos específico. En cambio, para los estímulos contracausales, la situación es distinta: en ambos grupos, la mayoría de los completamientos inadecuados parece estar guiado por una tendencia causal. Incluso con la presencia de una marca lingüística explícita que indica lo contrario, los lectores eligen completar los textos causalmente. Esta podría ser uno de los efectos más fuertes de la causalidad por defecto: se llega incluso a desconocer la guía semántica explícita presentada por el ítem en función de cumplir una expectativa causal en el completamiento. Una vez más, este patrón se mantiene para ambos niveles de escolaridad por lo que la tendencia causal no puede ser adjudicada a dicha variable y esto refuerza la posibilidad de contar con una base común desde la cual desarrollar habilidades de lectoescritura.

Si pasamos a revisar los tiempos de procesamiento, es posible ver que el patrón general tendiente hacia la causalidad se mantiene, pero presenta algunas particularidades. En la condición sin partícula conectiva, para el grupo de alta escolaridad, los ítems que fueron completados causalmente no sólo mostraron menores tiempos para la elección de la respuesta sino menores tiempos de lectura. Este dato es interesante, ya que podría estar exhibiendo que la expectativa causal ya está presente en la construcción del modelo mental del texto durante la primera lectura y que el completamiento 


\section{Zunino - Causalidad y Contracausalidad}

causal no se decide una vez concluida la lectura del fragmento y presentadas las opciones -acorde a la hipótesis de procesamiento incremental (Traxler, Bybee y Pickering, 1997)-. Sin embargo, esta capacidad prospectiva o anticipatoria de construcción de un modelo mental -vinculada con un proceso más activo de comprensión- sí estaría condicionada por el nivel de escolarización y, específicamente, por el entrenamiento lector.

Para el grupo de baja escolaridad, por su parte, en ambas condiciones, las diferencias en los tiempos de completamiento solo fueron cercanas a la significatividad: aun con diferencias nominales notables, los contrastes no resultaron estadísticamente significativos ${ }^{11}$. Este dato mostraría que si bien la organización conceptual y la tendencia interpretativa hacia la causalidad no depende de la escolarización, la velocidad -eficiencia- de ese proceso durante la comprensión y producción de discursos sí puede ser entrenada: para la causalidad, la escolarización facilita el proceso aunque no el resultado final, disminuyendo el esfuerzo cognitivo puesto en juego durante el procesamiento lingüístico; para la contracausalidad, la escolarización permite la adecuada interpretación del discurso, aun cuando no pueda igualar al procesamiento causal, en términos de eficiencia del proceso.

\section{Experimento 2}

Como adelantamos en la Introducción, para tener un panorama más cabal del proceso de producción de relaciones semánticas, específicamente el rol de la causalidad/contracausalidad, es necesario articular los datos presentados en el Experimento 1 con los resultados surgidos de un experimento que no restrinja el completamiento con opciones múltiples y nos permita analizar la producción espontánea de los sujetos. Así, nuestro segundo experimento intenta verificar: a) qué sucede cuando la tarea permite un completamiento espontáneo abierto; b) qué sucede con distintas construcciones linguiísticas y las partículas conectivas asociadas- que permiten verbalizar relaciones causales y contracausales. 
REMIE - Multidisciplinary Journal of Educational Research 273

\section{Método}

\section{Participantes}

En el grupo de alta escolaridad, participaron 34 sujetos hablantes nativos de español rioplatense -promedio de edad de 35,3 años; D.E.12,25-, con una escolarización formal de entre 12 y 18 años -promedio= 18,3; D.E.=0,95-. En el grupo de baja escolaridad, participaron 34 sujetos hablantes nativos de español rioplatense -promedio de edad de 26,1 años; D.E.= 12,2-, con una escolarización formal de entre 7 y 12 años -promedio=10,8; D.E. 0,7-. Dentro de cada grupo, los participantes se organizaron del mismo modo que en el Experimento 1. Con esta distribución, se obtuvieron datos de 17 participantes por condición evaluada.

\section{Materiales}

Se presentaron 20 fragmentos textuales conformados por dos cláusulas con la última proposición incompleta. De los 20 ítems, 10 de ellos eran causales y 10 contracausales. A su vez, cada uno presentaba dos condiciones según la partícula conectiva utilizada: "entonces" y "porque" para causales; "pero" y "aunque" para contracausales. Se controló la extensión por cantidad de palabras. Todos los estímulos tenían entre 12 y 18 palabras, con las siguientes medias de palabras por ítem: a) condición con "entonces": 14,2; b) condición con "porque": 14,6; c) condición con "pero": 14,8; d) condición con "aunque": 14,4. Se incluyeron, además, 10 ítems de relleno (fillers) en los que el completamiento requerido no pertenecía a la dimensión causal/contracausal.

Las consideraciones sobre estructura sintáctica y selección léxica y las particularidades metodológicas fueron las mismas que las descriptas para el Experimento1.

Ejemplos de estímulos utilizados:

(2) Mariana tiene un bebé recién nacido. Hacía horas que el bebito lloraba sin parar, entonces...

(3) Hace bastante que la computadora de Mario anda mal. Hoy decidió llevarla al servicio técnico porque... 


\section{Zunino - Causalidad y Contracausalidad}

(4) Gustavo y su mujer se reparten las tareas de la casa. Gustavo odia planchar, pero...

(5) A Carmen le encanta viajar. Aunque este año ya usó todas sus vacaciones, ...

(6) Mientras Julia estaba en el consultorio del dentista, su mamá....

\section{Procedimiento}

Este experimento fue programado en SuperLab 4.0. Se evaluaron tanto el tipo de respuesta, como los tiempos de iniciación verbal (TRI). La administración de la prueba fue individual. El procedimiento de presentación de consignas fue el mismo que en el Experimento 1.

El participante debía leer el estímulo presentado e, inmediatamente, producir la continuación de la segunda oración de modo oral. Todas las respuestas fueron grabadas para luego realizar una clasificación exhaustiva y adecuada de los completamientos espontáneos.

Se organizaron dos listas de 30 ítems: 10 targets causales, 10 targets contracausales y 10 fillers. Los estímulos se presentaron al azar.

\section{Resultados}

En primera instancia, se realizó una clasificación de los tipos de completamiento, en función de sistematizar el análisis. A partir de analizar todas las respuestas, la clasificación quedó conformada por siete tipos de respuesta para cada dimensión semántica. Para causales: 1) adecuada causal fuerte: causa-consecuencia directa; 2) adecuada causal débil: causaconsecuencia indirecta, premisa intermedia de una cadena causal mayor; 3) adecuada coherencia dudosa; 4) inadecuada contracausal; 5) inadecuada por cambio de estructura; 6) inadecuada incoherente; 7) tautológico/no informativo. Para contracausales: 1) adecuada contracausal; 2) adecuada no contracausal o de contraste genérico; 3) adecuada de coherencia dudosa; 4) inadecuada causal; 5) inadecuada cambio de estructura ${ }^{12}$; 6) inadecuada incoherente.

Se llevó a cabo un análisis exploratorio sobre los tiempos de iniciación verbal (TRI) y se depuró la base de datos crudos con el mismo método que en el experimento anterior (Cousineau \& Chartier, 2010; Ratcliff, 1979, 


\section{REMIE - Multidisciplinary Journal of Educational Research 275}

1993; Thompson, 2006): sólo se detectaron 5 casos extremos. Para poder analizar el tipo de respuesta con análisis de varianzas, se realizó una transformación logística sobre la proporción de respuestas de cada sujeto para los completamientos que interesaban especialmente en este trabajo: causal fuerte y contracausal -respuestas tipo 1 para cada dimensión-.

Tabla 4

Experimento 2: porcentajes totales y tiempos de iniciación verbal por tipo de completamiento.

\begin{tabular}{llllllllll}
\hline & & $1(\%)$ & $2(\%)$ & $3(\%)$ & $4(\%)$ & $5(\%)$ & $6(\%)$ & $7(\%)$ & TRI $(\mathrm{ms})(\mathrm{DE})$ \\
\hline \multirow{2}{*}{ ENTONCES } & AE & 72,4 & 24,1 & 0,6 & --- & --- & 2,4 & 0,6 & $6940,66(786,63)$ \\
& BE & 73,6 & 15,3 & 6,1 & -- & 1,2 & 0,6 & 1,8 & $7360,19(784,46)$ \\
PORQUE & AE & 67,7 & 25,7 & 2,4 & 0,6 & --- & 0,6 & 3,0 & $6973,97(692,35)$ \\
& BERO & 57,4 & 25,3 & 4,9 & --- & --- & 3,6 & 8,6 & $7135,34(612,29)$ \\
& AE & 71,6 & 15,4 & 5,9 & 3,6 & --- & 3,6 & --- & $8220,39(1434,73)$ \\
\multirow{4}{*}{ AUNQUE } & BE & 58,1 & 23,4 & 4,8 & 7,8 & 1,8 & 3,0 & 1,2 & $7863,46(2141,78)$ \\
& AE & 80,6 & 3,5 & 2,4 & 4,7 & 6,5 & 2,4 & --- & $7780,63(1348,97)$ \\
& BE & 52,7 & 7,8 & 5,4 & 6,6 & 25,7 & 1,2 & 0,6 & $8352,19(2136,84)$ \\
\hline
\end{tabular}

Elaboración propia. $\mathrm{AE}=$ Alta escolaridad; $\mathrm{BE}=\mathrm{B}$ aja escolaridad; Comp=completamiento.

Los números corresponden con la clasificación descripta al inicia de este apartado.

Tabla 5

Experimento 2: Tiempos de iniciación verbal (TRI) y desvios estándar (DE) por tipo de estímulo para completamientos 1.

\begin{tabular}{llll}
\hline & & Rtas TLog $(\mathrm{DE})$ & TRI $(\mathrm{ms})(\mathrm{DE})$ \\
\hline \multirow{2}{*}{ ENTONCES } & $\mathrm{AE}$ & $1,44(1,75)$ & $6940,66(786,63)$ \\
& $\mathrm{BE}$ & $1,71(2,46)$ & $7360,19(784,46)$ \\
\multirow{3}{*}{ PERO } & $\mathrm{AE}$ & $0,82(0,59)$ & $6973,97(692,35)$ \\
& $\mathrm{BE}$ & $0,24(0,48)$ & $7135,34(612,29)$ \\
\multirow{2}{*}{ AUNQUE } & $\mathrm{AE}$ & $1,38(1,72)$ & $8220,39(1434,73)$ \\
& $\mathrm{BE}$ & $0,31(0,61)$ & $7863,46(2141,78)$ \\
& $\mathrm{AE}$ & $2,72(2,95)$ & $7780,63(1348,97)$ \\
\hline
\end{tabular}

Elaboración propia. $\mathrm{AE}=$ Alta escolaridad; $\mathrm{BE}=$ Baja escolaridad; $\mathrm{TL}$ gotransformación logística; TRI=tiempo de iniciación verbal; $\mathrm{DE}=$ desvío estándar; ms=milisegundos. 
En primer lugar, se realizó un análisis factorial completo con un factor entre sujetos -ESCOLARIDAD- y un factor intra-sujeto -CONECTOR-, considerando los completamientos de tipo 1 para cada dimensión. Para la medida de tipo de respuesta, se halló efecto principal significativo para el factor entre sujetos ESCOLARIDAD $\left(\mathrm{F}_{(1,30)}=8,13 ; \mathrm{p}=, 008\right)$. También resultó significativa la interacción CONECTOR $*$ ESCOLARIDAD $\left(\mathrm{F}_{(1,30)}=5,25\right.$; $\mathrm{p}=0,29)$. Por su parte, para la medida de tiempo de iniciación verbal en los casos de completamiento de tipo 1, sólo se halló efecto principal de la variable CONECTOR $\left(\mathrm{F}_{(1,30)}=7,06 ; \mathrm{p}=, 013\right)$; el factor ESCOLARIDAD y la interacción entre ambos no resultaron significativos.

En segunda instancia, se realizaron varias comparaciones específicas por ANOVA de medidas repetidas, para cada grupo de escolaridad: 1) completamiento causal con "entonces" vs. con "porque"; 2) completamiento contracausal con "pero" vs. con "aunque"; 3) completamiento causal de mejor rendimiento (con "entonces" para ambos grupos) vs. contracausal de mejor rendimiento (con "aunque" para AE y "pero" para BE); 4) causales vs. contracausales. Para todos los contrastes, se calculó F1 y F2 y las comparaciones múltiples fueron tratadas con corrección de Bonferroni.

1) Si bien los completamientos de consecuencias resultaron más sencillos para ambos grupos, la diferencia respecto de los completamientos de causas es estadísticamente significativa sólo para el grupo $\mathrm{BE}(\mathrm{F}(1,15)=5,92$; $\mathrm{p}=$,028). Las diferencias en los TRI, sin embargo, no mostraron ventajas significativas para ninguna de las dos condiciones en ninguno de los dos grupos de participantes.

2) Si bien en el grupo AE existe una ventaja de la condición con "aunque" tanto en el tipo de completamiento como en el tiempo requerido, sólo el contraste $F 1$ de la medida TRI resultó significativo $(F(1,16)=9,49$; $\mathrm{p}=, 007)$. Para el grupo BE, ninguno de los contrastes resultó significativo.

3) Ambos grupos difirieron respecto de qué condición mostró el mejor rendimiento en la dimensión contracausal, por lo que los contrastes realizados fueron distintos. Para el grupo AE, las dos condiciones de mejor rendimiento fueron "entonces" y "aunque". Los tipos de respuesta, mostraron una ventaja significativa de la condición de mejor rendimiento contracausal $(\mathrm{F}(1,16)=5,08, \mathrm{p}=, 039)$. Para el tiempo de iniciación verbal, en 
cambio, la ventaja fue significativa pero a favor de la condición causal $(\mathrm{F} 1(1,16)=5,91, \mathrm{p}=, 027 ; \mathrm{F} 2(3,39)=7,24, \mathrm{p}=, 001)$. Por su parte, para el grupo BE, el mejor rendimiento se halló con "entonces" y "pero". La medida de tipo de completamiento exhibió un contraste estadísticamente significativo a favor de la condición causal $(\mathrm{F}(1,16)=4,38, \mathrm{p}=, 05)$; mientras que la medida de tiempo no arrojó contrastes significativos.

4) Si se toma la totalidad de los ítems causales y contracausales -sin importar el conector utilizado en cada dimensión-, la diferencia en la cantidad de completamientos adecuados -respuesta tipo 1- de cada dimensión no resulta significativa, pero la ventaja en los TRI para ítems causales sí es estadísticamente significativa: $\mathrm{F}(1,19)=5,6 ; \mathrm{p}=, 025$.

Finalmente, se hicieron los contrastes entre grupos (AE vs. BE):

1) completamiento 1 con "entonces": el contraste sobre tipo de respuesta no fue estadísticamente significativo, sí, en cambio, el realizado sobre TRI, que mostró una ventaja para el grupo $\mathrm{AE}(\mathrm{F} 1(1,31)=4,93 ; \mathrm{p}=, 034$; $\mathrm{F} 2(1,19)=4,94 ; \mathrm{p}=, 039)$.

2) completamiento 1 con "porque": se halló significatividad estadística a favor del grupo AE para el contraste por tipo de respuesta $(F(1,31)=9,46$; $\mathrm{p}=, 004)$, no así para la medida de tiempo.

3) completamiento 1 con "pero": el contraste por tipo de respuesta resultó estadísticamente significativo a favor del grupo $\mathrm{AE}(\mathrm{F}(1,31)=5,78 ; \mathrm{p}=, 022)$, pero las diferencias en tiempo no mostraron significatividad estadística entre los grupos.

4) completamiento 1 con "aunque": sólo se halló una diferencia estadísticamente significativa en la comparación por tipo de respuesta, también a favor del grupo $\mathrm{AE}(\mathrm{F}(1,31)=10,22 ; \mathrm{p}=, 003)$.

\section{Discusión}

En primera instancia, diremos que encontramos una ventaja causal cuando comparamos las dos dimensiones de modo global. Si bien la diferencia en la cantidad de completamientos considerados causales y contracausales de tipo 1 no es significativa, sí hay una diferencia significativa en los tiempos de completamiento. Esto es, resulta más sencillo completar relaciones semánticas dentro de la dimensión causal -en cualquiera de sus formas 
estructurales- que en la dimensión contracausal, y esta ventaja se exhibe especialmente en los tiempos requeridos para producir el segmento faltante de la relación.

Existe, además, una interacción entre la ESCOLARIZACIÓN y la DIMENSIÓN SEMÁNTICA analizada. Este patrón parece mostrar que la escolarización vuelve a modular la marcada ventaja causal que se presentaría por defecto. La diferencia entre grupos respecto de los completamientos considerados contracausales es siempre significativa y la brecha es marcadamente superior que para los causales: construir contracausalidad es más complejo siempre, pero, además, está significativamente condicionado por el nivel de escolarización formal del sujeto. No así los tiempos requeridos para producir ese completamiento: en los casos en que se logra una producción adecuada, el proceso on-line no difiere entre los grupos. Existe, además, otro dato importante a tener en cuenta que confluye para respaldar este patrón: exclusivamente en el grupo BE encontramos más de un $25 \%$ de completamientos que fuerzan la estructura del ítem -respuestas de tipo 5: ver nota 12-. Aquí se vería nuevamente un efecto máximo de esta tendencia causal: los sujetos buscan completar la relación con consecuencias a expensas de modificar la estructura del estímulo dado, tal como sucedía en el Experimento 1 cuando desconocían la guía semántica otorgada por la partícula conectiva para elegir la opción causal.

Dentro de la dimensión contracausal, la condición con "aunque" sigue mostrando una ventaja en el grupo AE, pero no resulta significativa. Para el grupo BE, por su parte, es la condición con "pero" la que muestra mejor rendimiento -en todas las medidas-, aunque ninguno de los contrastes llega a mostrar significatividad estadística. En términos explicativos, creemos que esta diferencia entre grupos puede comprenderse en función de las características semánticas propias de cada partícula conectiva en español. "Pero" tiene una amplitud semántica mayor y suele utilizarse para relaciones de contraste genérico -de hecho, alrededor del $23 \%$ de los completamientos con "pero" fueron clasificados como contrastes amplios para el grupo $\mathrm{BE}^{13}$.

A partir de estos datos, entendemos que las posibilidades de precisar el completamiento desde el contraste genérico hacia la contracausalidad propiamente dicha serían directamente proporcionales al nivel de escolarización $^{14}$. Por otro lado, el dato ya mencionado sobre el alto 
porcentaje de modificaciones de estructura que mostró la condición con "aunque" en el grupo BE, que lleva el estímulo desde una construcción concesiva hacia una adversativa en función de producir completamiento causal, podría entenderse en el mismo sentido: en esos casos, los sujetos procesaron, encadenadamente, «contraste genérico» + «causalidad». Ambas partes de ese patrón de procesamiento parecen ser influidas por la escolarización: los sujetos de $\mathrm{AE}$ procesan con mayor precisión la contracausalidad propiamente dicha sin tender al contraste genérico y pueden modular su tendencia causal por defecto cuando la marca semántica explícita es precisa en tanto instrucción de procesamiento.

Por último, para la dimensión causal es interesante notar que el peso de la condición que consideramos más sencilla, no marcada o por defecto -con "entonces" en orden habitual [causa]-[efecto]- es la que muestra ventajas en ambos grupos de sujetos, e incluso los iguala entre sí en términos de porcentaje de completamientos adecuados. Parece haber una especial influencia de esta estructura, que se traduce en una mayor facilidad para construir relaciones causales a partir de completar «consecuencias» en lugar de «causas», como patrón transversal no condicionado por la escolarización formal. Sí existe, sin embargo, una diferencia significativa en la velocidad requerida para ese completamiento en cada uno de los grupos y esta diferencia se articula con la condición particular analizada: mientras los sujetos de mayor escolaridad requieren significativamente menos tiempo que los de menor escolaridad para completar «consecuencias», cuando se trata de producir «causas», ese patrón se invierte y el primer grupo sí mejora significativamente en términos del tipo de completamiento, pero lo logra sólo a expensas de un proceso online más costoso que lo asimila al segundo grupo. Es posible pensar aquí también otro efecto modulador de la escolarización, en este caso, dentro de la dimensión causal: para la construcción/producción de relaciones causales la estructura en orden de iconicidad invertida "[efecto] porque [causa]" no sería la estructura por defecto -o primitiva- común a todos los hablantes, sino que sería una construcción derivada de la versión icónica y, por ende, susceptible de entrenamiento.

En síntesis, en esta tarea parece exhibirse con máxima claridad la escala de dificultad de procesamiento que también se ha hallado en trabajos sobre 
comprensión (Zunino, 2014; Zunino, Abusamra \& Raiter, 2016) y que sólo podíamos observar preliminarmente en el Experimento 1 dado que no contábamos con estructuras causales invertidas del tipo "[efecto] porque [causa]" ni formas contracausales con estructura concesiva. La estructura causal en orden habitual [causa]-[efecto] sería la construcción más accesible y la que se procesaría por defecto, seguida por la estructura causal en orden invertido [efecto]- [causa] y, por último, las relaciones que suspenden una causalidad esperada o contracausales. Esa gradación se sostiene no sólo a partir de los resultados dentro de cada grupo de sujetos sino a través de mostrar que el efecto de la escolarización se exhibe más claramente en aquellas construcciones que quiebran alguna característica respecto de la considerada básica o primitiva: relaciones causales en orden habitual [causa]- [efecto].

\section{Discusión General}

Si bien este trabajo se enmarca en los principios y perspectiva epistemológica de la psicolingüística como interdisciplina experimental (De Vega \& Cuetos, 1999; Jääskeläinen, 2012), nuestro objetivo principal para el análisis de los datos es comprender y reflexionar sobre cuáles de esos datos pueden ser susceptibles de transferencia al ámbito educativo y sobre qué propuestas son posibles en términos de una aplicación pedagógica concreta.

Específicamente, en el intento de suplir algunas vacancias entre los estudios de procesamiento de discurso y relaciones semánticas (Zunino, 2014, 2016; Degand \& Sanders, 2002; Goldman et al., 1999; Graesser, Millis \& Zwaan, 1997; Graesser et al., 2003; Kendeou \& van den Broek 2007; Kintsch \& Van Dijk, 1978; Koornneef \& van Berkum, 2006; McNamara et al. 1996; Molinari Marotto \& Duarte, 2007; Murray, 1997; Noordman et al., 1992; Noordman \& Vonk, 1998; Noordman et al., 2015; Martins et al., 2006; Ozuru et al., 2009; Sanders, 2005; Segal, Duchan \& Scott, 1991; Simner \& Pickering, 2005; Pickering \& Majid, 2007; Portolés, 1998; Van den Broek, Linzie, Fletcher \& Marsolek, 2000; Xiang \& Kuperberg, 2015; Zwaan \& Radvansky, 1998), nos interesaba analizar: a) de qué modo la escolarización formal interviene en la producción de relaciones causales y contracausales; b) comparar estos datos con aquellos hallados para tareas de comprensión; c) analizar qué pueden 
aportar estos resultados para mejorar los procesos de alfabetización en distintos niveles educativos.

Los complejos procesos de comprensión y producción de discursos, tanto escritos como orales, son centrales para posibilitar y potenciar los procesos de aprendizaje, sobre todo en los niveles secundario y superior. En ese sentido, comprender qué aspectos de aquellos procesos no resultan dependientes del entrenamiento y cuáles sí, parece decisivo para diseñar estrategias eficaces que utilizar capacidades primitivas compartidas por todos los hablantes como estructura de base para proyectar y desarrollar otras habilidades.

Específicamente para el procesamiento de relaciones semánticas, componente central para la comprensión y producción de discursos orales y escritos (Zunino, 2014; Segal et al., 1991; van Dijk, 1992; Zwaan \& Radwansky, 1998; entre mucho otros), hay tres cuestiones especialmente importantes a tener en cuenta a la hora de pensar modos de intervención en el espacio educativo: a) cómo interviene el conocimiento linguiístico, específicamente las instrucciones semánticas como los conectores, durante el procesamiento; b) de qué modo esto se articula con el bagaje de conocimiento previo que el sujeto puede poner en juego y la organización conceptual de ese conocimiento; c) observar si existen dimensiones conceptuales/semánticas preferidas, que exhiben mayor facilidad de procesamiento. Todos estos datos permitirían, por un lado, preparar materiales didácticos especialmente controlados respecto de estos aspectos (psico)lingüísticos, y por otro, intervenir en poblaciones disímiles con herramientas pedagógicas disímiles, que potencien las habilidades de cada grupo y logren, al final del proceso, un resultado común a pesar de posibles diferencias en el punto de partida.

Nuestro primer experimento buscaba analizar si existía ventaja causal en la construcción de coherencia y posterior completamiento de un fragmento textual y de qué modo intervenía la presencia de una partícula conectiva en este proceso. Los resultados nos permitieron observar que:

1) las relaciones causales muestran una ventaja significativa en ambas condiciones -sin y con partícula conectiva-;

2) este patrón de tendencia causal no resultó dependiente de factores culturales como el nivel de escolarización; 


\section{Zunino - Causalidad y Contracausalidad}

3) en ocasiones, la preferencia por establecer completamientos causales se impuso sobre la indicación -restricción- semántica establecida por la partícula conectiva específica.

Por su parte, nuestro segundo experimento, intentaba especificar el patrón hallado en el primero, analizando distintas estructuras linguiísticas para verbalizar las mismas relaciones semánticas, y evaluar la tarea con un paradigma de completamiento espontáneo, que no restringiera las opciones $a$ priori. Nuestros datos mostraron que:

1) es más sencillo producir/completar relaciones causales que contracausales;

2) la escolarización modula la brecha -ventaja causal- entre ambas dimensiones;

3) la escolarización no condiciona los tiempos del proceso cuando el sujeto llega a un completamiento semánticamente consistente;

4) el patrón corresponde a un continuum de complejidad discutido para la comprensión de este tipo de relaciones (Zunino, 2014; Zunino et al., 2016) -causales con "entonces" vs. "porque"; contracuasales con "pero" vs. "aunque"- también se exhibe en tareas de producción y se encuentra significativamente condicionado por la escolarización: la estructura por defecto correspondería a las causales en orden icónico "[causa]-[efecto]", lo que aportaría evidencia para hallar componentes comunes a los procesos de comprensión y producción de discurso (Mar, 2004).

Resultados previos en tareas de comprensión (Zunino, 2014, 2016; Zunino \& Raiter, 2012) mostraron, en términos generales, que: a) la presencia de marca semántica explícita es facilitadora en todas las relaciones y en todos los grupos de sujetos; sin embargo, su efecto es mayor cuanto mayor sea la dificultad del material lingüístico a procesar, que, a su vez, es el que más entrenamiento requiere por ser el que no se procesa por defecto; b) el procesamiento de relaciones causales, centrales para la coherencia discursiva/textual, exhibe una ventaja significativa respecto de relaciones semánticas que quiebran esa causalidad esperada como estrategia interpretativa por defecto en todos los grupos, por lo que no parece estar condicionada por el entrenamiento; c) los modos específicos en que se observa esa ventaja causal durante el procesamiento de lenguaje podría estar indicando una organización 


\section{REMIE - Multidisciplinary Journal of Educational Research 283}

causal de nuestras representaciones mentales; más específicamente, una organización causal con estructura icónica causa-efecto. Los resultados exhibidos en este trabajo centrado en el proceso de producción de relaciones semánticas en el discurso acompañan y refuerzan este patrón, lo que permite llegar a conclusiones más certeras y pensar estrategias didácticas más globales.

De modo general, estos datos muestran que siempre sería preferible trabajar con textos/discursos que presenten marcas semánticas explícitas como las partículas conectivas, en tanto éstas pertenecen al conocimiento linguístico común que manejan todos los hablantes -no variable con el grado de experticia- y muchas veces pueden ser las únicas pistas de las que el estudiante dispone para comprender cómo se vincula la información que se le presenta, para así poder construir un modelo mental coherente (Zunino et al., 2016). Este punto es central e incumbe a todos los materiales didácticos utilizados en todos los niveles educativos: un texto con conectores y marcadores del discurso explícitos y específicos propicia una mejor comprensión de las relaciones de significado presentadas por el texto y una más exitosa comprensión global. Si bien para la causalidad, que parece ser procesada por defecto, no resultan determinantes, pero tampoco obstaculizadores; en cambio, sí son decisivos para el procesamiento de contracausalidad.

Específicamente respecto del procesamiento semántico, podemos decir que si se acepta que la organización conceptual causal es transversal a todos los sujetos, y que el procesamiento de causalidad resulta una tendencia por defecto, el trabajo pedagógico centrado en relaciones causales podría ser un mecanismo eficaz que aproveche productivamente habilidades ya dadas, y comunes a todos los sujetos, para desarrollar otras capacidades sí dependientes del entrenamiento o de procesos de aprendizaje explícitos. Con esto planteamos que tanto los materiales didácticos como los abordajes pedagógicos pueden hacer un especial uso de plantear textuales y discursos con fuerte impronta causal: estos vínculos podrían ser comprendidos por la totalidad de los estudiantes, sin que diferencias en los bagajes previos resulten determinantes. Una vez planteada una situación problemática -del tipo que sea- en estos términos, y haciendo anclaje en este esqueleto causal, se podría trabajar sobre otros vínculos como temporales, condicionales, espaciales, u otras relaciones lógicas en general. Se ve así que esta propuesta trasciende el trabajo pedagógico en el área específica de lengua y literatura, ya que se trata de una 


\section{Zunino - Causalidad y Contracausalidad}

perspectiva decisiva para abordar cualquier nuevo texto e incluso discursos orales propios del ámbito áulico en cualquier asignatura. Por otra parte, creemos que nuestros resultados sobre el proceso de producción de relaciones semánticas en el nivel discursivo podrían ser útiles para desarrollar estrategias de mejoramiento de las habilidades retóricas y de exposición oral de los estudiantes de niveles secundario, terciario y universitario, que suelen carecer de recursos orales a la altura de lo que se les requiere durante su recorrido académico.

Proponemos así un potencial círculo virtuoso en el que la causalidad resulte un andamiaje semántico/conceptual para adquirir conocimientos nuevos a través de textos/discursos, para luego construir y comprender nuevas relaciones de significado sobre esa información, en pos de, finalmente, poder producir nuevos textos/discursos articulando nuevas relaciones semánticas -causales y de todo tipo- entre conocimientos recientemente adquiridos y aquellos previamente almacenados. Creemos que de esto debemos hablar cuando hablamos de aprendizaje.

\section{Notas}

${ }^{1}$ Involucrar este elemento no es sencillo porque, entre otras cosas, aún está en discusión qué se entiende por "conocimiento de mundo". Sin embargo, es, sin dudas, una variable que no puede desatenderse en el estudio de la comprensión de discurso (Noordman \& Vonk, 1998; Graesser, McNamara \& Louwerse, 2003; Raiter, 2003; entre otros).

${ }^{2}$ Para el análisis del contenido semántico de los textos, Kintsch y Van Dijk (1978) proponen la identificación de dos niveles (previos a la construcción de y con menor abstracción que el modelo de situación): texto de superficie (unidades lingüísticas simples) y texto base proposicional (proposiciones complejas, construidas a partir de unidades más simples, que conforman una nueva unidad de significado). Este último se describe como un nivel de mayor abstracción que el primero: el texto base es la representación proposicional del texto de superficie.

${ }^{3}$ Para el caso del Experimento 1, parte del cual ha sido publicado recientemente, se consignará la referencia bibliográfica correspondiente en cada una de las Tablas de resultados.

${ }^{4}$ Este grupo de informantes fue utilizado en el estudio recientemente publicado en Zunino et al. (2016).

${ }^{5}$ Los mismos materiales fueron utilizados en el experimento publicado recientemente en Zunino et al. (2016). 


\section{REMIE - Multidisciplinary Journal of Educational Research 285}

${ }^{6}$ Se utilizó el mismo procedimiento que en experimento publicado recientemente en Zunino (2016).

${ }^{7}$ Los datos presentados en el Experimento 1 de este trabajo corresponden a los publicados recientemente en Zunino (2016). En esta oportunidad se busca articular esta primera evidencia con nuevos resultados de un segundo experimentos y realizar los análisis con otro objetivo central: su transferencia al ámbito educativo.

${ }^{8}$ Se considera que estos análisis pueden eliminar entre el 1 y el $10 \%$ de la totalidad de los datos de una muestra.

${ }^{9}$ Para todas las comparaciones múltiples se utilizó corrección de Bonferroni.

${ }^{10}$ Para una discusión sobre procesos potencialmente "activos" y "pasivos" durante la lectura ver Degand, Lefévre y Bestgen (1999); McNamara et al. (1996), entre otros.

${ }^{11}$ Esta situación está ligada a los valores de los desvíos estándar. El grupo de baja escolaridad, parece mostrar menor homogeneidad entre los sujetos y, por ende, los desvíos estándar son muy grandes: las pruebas estadísticas de comparación de medias -análisis de varianza- son altamente sensibles a las distribuciones con excesiva variación. La mayor variabilidad puede ocultar diferencias estadísticamente significativas en las comparaciones.

${ }^{12}$ Se da especialmente con ítems con "aunque", que, en español, permiten construir tanto estructuras concesivas como adversativas. Hablamos de casos como "Romina tenía ganas de hacer una torta de manzanas. Aunque le faltaban algunos ingredientes, ... entonces los fue a comprar."

${ }^{13}$ Casos como "Es feo, pero simpático." (aunque esto podría ser entendido como una cadena causal-contracausal abreviada; ver nota 5) u "Hoy hay sol, pero mañana va a llover." frente a casos contracausales como "Marcos no regó las plantas, pero no se secaron." (que suspende la relación causal esperada "Marcos no regó las plantas, entonces se secaron.”).

${ }^{14}$ Un patrón similar se halla en tareas de comprensión (ver Zunino, 2016).

\section{Referencias}

Cheng, P. W. (1997). From covariation to causation: A causal power theory. Psychological Review, 104, 367-405. doi: 10.1037//0033295X.104.2.367

Couper-Kuhlen, E., \& Kortmann, B. (Eds.). (2000). Cause, condition, concession, contrast. Cognitive and Discoursive Perspectives. Berlin: Mouton de Gruyter. 
Cousineau, D., \& Chartier, S. (2010). Outliers Detection and Treatment: A review. International Journal of Psychological Research, 3(1), 58-67. Recuperado de http://revistas.usb.edu.co/index.php/IJPR/article/view/844/601

Davidson, D. (1985). Essays on Actions and Events. Oxford: Clarendon Press. De Vega, M., \& Cuetos, F. (1999). Psicolingüística del español. Madrid: Trotta.

Degand, L., \& Sanders, T. (2002). The impact of relational markers on expository text comprehension text comprehension in L1 and L2. Reading and Writing, 15 (7-8), 739-758. doi:

10.1023/A:1020932715838

Degand, L., Lefèvre, N., \& Bestgen, Y. (1999). The impact of connectives and anaphoric expressions on expository discourse comprehension.

Document Design, 1, 39-51. doi: 10.1075/dd.1.1.06deg

Dery, J. E., \& Koenig, J. P. (2015). A Narrative-Expectation Based Approach to Temporal Update in Discourse Comprehension. Discourse Processes, 52(7), 559-584. doi: 10.1080/0163853X.2014.966293

Goldman, S., Graesser, A., \& van den Broek, P. (1999). Narrative Comprehension, Causality, and Coherence. Essays in Honor of Tom Trabasso. Londres: Lawrence Erlbaum.

Goldvarg, E., \& Johnson-Laird, P. N. (2001). Naive causality: a mental model theory of causal meaning and reasoning. Cognitive Science, 25, 565-610. doi: 10.1207/s15516709cog2504_3

Graesser, A., McNamara, D., \& Louwerse, M. (2003). What do readers need to learn in order to process coherence relations in narrative and expository text? En A. Sweet y C. Snow (Eds.). Rethinking reading comprehension (pp. 82-98). Nueva York: Guilford.

Graesser, A., Millis, K., \& Zwaan, R. (1997). Discourse Comprehension. Annual Review Psychology, 48: 163-189. doi:

10.1146/annurev.psych.48.1.163

Gravetter, F., \& Wallnau, L. (2009). Statistics for the behavioral sciences. Belmont, California: Wadsworth.

Hume, D. (1740/1878). A Treatise of Human Nature. Oxford: Oxford University Press. 
Jääskeläinen, L. P. (2012). Introduction to Cognitive Neurosciene. Ventus Publishing.

Kant, I. (1787/2008). Crítica de la razón pura. Madrid: Taurus.

Kendeou, P., \& van den Broek, P. (2007). The effects of prior knowledge and text structure on comprehension processes during reading of scientific texts. Memory and Cognition, 35(7), 1567-1577. doi:

10.3758/BF03193491

Kim, J. (2007). Causation and Mental Causation. En B. McLaughlin y J. Cohen (Eds.) Contemporary debates in philosophy of mind (pp.227-243).

Singapur: Blackwell.

Kintsch, W., \& Van Dijk, T. (1978). Toward a model of text comprehension and production. Psychlogical Review, 85(5), 363-394. doi:

10.1037/0033-295X.85.5.363

Koornneef, A.W., \& Van Berkum, J. J. A. (2006). On the use of verb-based implicit causality in sentence comprehension: Evidence from self-paced reading and eye tracking. Journal of Memory and Language, 54, 445465. doi: 10.1016/j.jml.2005.12.003

León, J., \& Peñalba, G. (2002). Understanding Causality and Temporal Sequence in Scientific Discourse. En J. Otero, J. León y A. Graesser (Eds). The Psychology of Science Text Comprehension (pp. 155-178). Nueva Jersey: Lawrence Erlbaum.

Leslie, A. (1994). ToMM, ToBY y Agencia: arquitectura básica y especificidad de dominio. En L. Hirschfeld, L. y S. Gelman (Eds.). Cartografía de la mente. La especificidad de dominio en la cognición y en la cultura (pp. 177-216). Barcelona: Gedisa.

Mar, R. A. (2004). The neuropsychology of narrative: story comprehension, story production and their interrelation. Neuropsychologia, 42, 14141434. doi: 10.1016/j.neuropsychologia.2003.12.016

Martins, D., Kigiel, D., \& Jhean-Larose, S. (2006). Influence of Expertise, Coherence, and Causal Connectives on Comprehension and Recall of an Expository Text. Current psychology letters, 20 (3), 1-13. Recuperado de http://cpl.revues.org/1146

McNamara, D., Kintsch, E., Songer, N., \& Kintsch, W. (1996). Are Good Texts Always Better? Interactions of Text Coherence, Background Knowledge, and Levels of Understanding in Learning from Text. 
Cognition and Instruction, 14(1), 1-43. doi:

10.1207/s1532690xci1401_1

Michotte, A. (1963). The perception of causality. Andover: Methuen.

Molinari Marotto, C., \& Duarte, A. (2007). Comprensión del texto narrativo e inferencias. Subjetividad y procesos cognitivos, 163-183. Recuperado de http://dspace.uces.edu.ar:8180/xmlui/handle/123456789/136

Murray, J. D. (1997). Connectives and narrative text: The role of continuity. Memory \& Cognition, 25(2), 227-236. doi: 10.3758/BF03201114

Noordman, L., \& Vonk, W. (1998). Memory-based processing in understanding casual information. Discourse Processes, 26(2-3), 191212. doi: 10.1080/01638539809545044

Noordman, L., Vonk, W., \& Kempff, H. F. (1992). Causal inferences during the reading of expository text. Journal of Memory and Language, 31, 573-590. doi: 10.1016/0749-596X(92)90029-W

Noordman, L. G. M., Vonk, W., Cozijn, R., \& Frank, S. (2015). Causal inferences and world knowledge. In E. J. O'Brien, A. E. Cook, \& R. F. Lorch (Eds.), Inferences during reading (pp. 260-289). Cambridge, UK: Cambridge University Press.

Ozuru, Y., Dempsey, K., \& McNamara, D. S. (2009). Prior knowledge, reading skill, and text cohesion in the comprehension of sciences texts. Learning and Instruction, 19, 228-242. doi:

10.1016/j.learninstruc.2008.04.003

Pérez, D. (2009). Conceptos fenoménicos, conceptos psicológicos y la explicación de la conciencia. Revista Hispanoamericana de Filosofía, 41(121), 85-97. Recuperado de http://critica.filosoficas.unam.mx/pg/en/descarga.php?id_volumen=15\&i d_articulo=76

Pickering, M., \& Majid, A. (2007). What are implicit causality and consequentiality? Language and Cognitive Processes, 22(5), 780-788. doi: 10.1080/01690960601119876

Portolés, J. (1998). Marcadores del discurso. Barcelona: Ariel.

Raiter, A. (2000). Las referencias (mentales) para establecer el significado lingüístico: conocimientos previos, memoria enciclopédica, sistema de creencias y sentido común. (Tesis Doctoral no publicada.) Facultad de Filosofía y Letras, Universidad de Buenos Aires, Buenos Aires. 
Raiter, A. (2003). Lenguaje y sentido común. Las bases para la formación del discurso dominante. Buenos Aires: Biblos.

Ratcliff, R. (1979). Group Reaction Time Distributions and Analysis of Distribution Statistics. Psychological Bulletin, 86, 446-461. doi: 10.1037/0033-2909.86.3.446

Ratcliff, R. (1993). Methods with Dealing with Reaction Time Outliers. Psychological Bulletin, 114 (3), 510-532. doi: 10.1037/00332909.114.3.510

Sanders, T. J. M. (2005). Coherence, Causality and Cognitive Complexity in Discourse. Proceedings/Actes SEM-05. First International Symposium on the Exploration and Modeling of Meaning, 105-114.

Schlottamnn, A. (2001). Perception versus Knowledge of Cause and Effect in Children: When Seeing in Believing. Current Directions in Psychological Science, 10(4), 111-115. doi: 10.1111/1467-8721.00128

Segal, E., Duchan, J., \& Scott, P. (1991). The Role of interclausal connectives in narrative structirung: Evidence from adults interpretations of simple stories. Discourse Processes, 14, 27-54. doi:

$10.1080 / 01638539109544773$

Simner, J., \& Pickering, M. J. (2005). Planning causes and consequences in discourse. Journal of Memory and Language. doi:

10.1016/j.jml.2004.04.006

Sloman, S. (2005). Casual Models. How People Think about the World and its Alternatives. New York: Oxford University Press.

Sperber, D., Premack, D., \& Premack, A. J. (Eds.). (1995) Causal Cognition: A Multidisciplinary Debate Nueva York: Oxford University Press.

Strawson, P. F. (1985). Causation and explanation. En B. Vermazen y M. Hintikka (edd.), Essays on Davidson: Actions and Events (pp.115-135), Oxford: University Press.

Thompson, G. (2006). An SPSS implementation of the nonrecursive outlier deletion procedure with shifting z score criterion (Van Selst \& Jolicoeur, 1994). Behavior Research Methods, 38(2), 344-352. Recuperado de http://citeseerx.ist.psu.edu/viewdoc/download?doi=10.1.1.471.5621\&rep $=$ rep1\&type $=$ pdf

Traxler, M., Bybee, M., \& Pickering, M. (1997). Influence of Connectives on Language Comprehension: Eye-tracking Evidence for Incremental 
Interpretation. The Quarterly Journal of Experimental Psychology, 50A (3), 481-497. doi: 10.1080/027249897391982

van den Broek, P., Linzie, B., Fletcher, C.R., \& Marsolek, C. J. (2000). The role of causal discourse structure in narrative writing. Memory \& Cognition, 28, 711-721. doi:10.3758/BF03198405

van Dijk, T. (1992). La ciencia del texto. Un enfoque interdisciplinario. Barcelona: Paidós.

van Dijk, T., \& Kintsch, W. (1983). Strategies of Discourse Comprehension. Nueva York: Academic Press.

van Selst, M., \& Jolicoeur, P. (1994). A solution to the effect of sample size on outlier elimination. The Quarterly Journal of Experimental Psychology, 47A, 631-650. doi: 10.1080/14640749408401131

Viale, R. (1999). Causal cognition and casual realism. International Studies in the Philosophy of Science, 2, 151-196. Recuperado de http://www.sociologia.unimib.it/ricerca/ricerca/pdfDownload.php?idPap er $=58$

Waldmann, M. (2001). Predictive versus diagnostic causal learning: Evidence from an overshadowing paradigm. Psychonomic Bulletin and Review, 8 (3), 600-608. doi: 10.3758/BF03196196

Woods, A., Fletcher, P., \& Hughes, A. (1986). Statistics in Language Studies. Cambridge: Cambridge University Press.

Xiang, M., \& Kuperberg, G. (2015). Reversing Expectations during Discourse Comprehension. Language, Cognition and Neuroscience, 30(6), 648672. doi: 10.1080/23273798.2014.995679

Zunino, G. M. (2014). Procesamiento psicolingüístico de relaciones semánticas: causalidad y contracausalidad. Tesis (Doctorado en Linguiística), Facultad de Filosofía y Letras, Universidad de Buenos Aires.

Zunino, G. M. (2016). Construcción de causalidad y contracausalidad en sujetos con distinto nivel educativo. Actas de Investigación Psicológica, 6(2), 2412-2421. doi: 10.1016/j.aipprr.2016.06.004

Zunino, G. M., Abusamra, V., \& Raiter, A. (2016). Causalidad, iconicidad y continuidad: el efecto del conocimiento previo sobre el mundo en la comprensión de relaciones causales. Alfa. Revista de Linguística, 60(2), 261-285. doi: 10.1590/1981-5794-1608-2 
REMIE - Multidisciplinary Journal of Educational Research 291

Zunino, G. M., \& Raiter, A. (2012). Construcción de coherencia textual. Un estudio preliminar acerca de la causalidad y sus implicancias neuropsicolingüísticas. Revista Neuropsicología Latinoamericana, 4(2), 1-15. doi: 10.5579/rnl.2012.0082

Zwaan, R., \& Radwansky, G. (1998). Situation Models in Language

Comprehension and Memory. Psychological Bulletin, 123, 162-185. doi: 10.1037/0033-2909.123.2.162

Zwaan, R., Magliano, J., \& Graesser, A. (1995). Dimensions of Situation Model Construction in Narrative Comprehension. Journal of Experimental Psychology, Learning, and Cognition, 21(2), 386-397. doi: 10.1037/0278-7393.21.2.386

Gabriela M. Zunino es Doctora en Lingüística por la Facultad de Filosofía y Letras de la Universidad de Buenos Aires.

Contacto: Universidad de Buenos Aires. Facultad de Filosofía y Letras. Puán 480, 1420 CABA, Argentina

E-mail: gmzunino@conicet.gov.ar 